\title{
Interactive Analysis of Terabyte-sized SEM-EDS Hyperspectral Images
}

\author{
Antoine Vandecreme ${ }^{1}$, Peter Bajcsy ${ }^{1}$, Nicholas W.M. Ritchie ${ }^{2}$, and John Henry J. Scott ${ }^{2}$ \\ ${ }^{1}$ Information Technology Lab, National Institute of Standards and Technology, Gaithersburg, MD. \\ ${ }^{2 .}$ Material Measurement Lab, National Institute of Standards and Technology, Gaithersburg, MD.
}

Scanning electron microscopy with energy-dispersive X-ray spectrometry (SEM-EDS) was used to characterize a macroscopic artifact from Peru (a Moche cast figure) [1]. A 3D hyperspectral datacube was acquired over 51.2 hours, measuring 11,520 x 9,984 pixels with 2,048 energy channels per pixel; each pixel corresponds to $900 \mathrm{~nm}$ in the $\mathrm{x}$ and $\mathrm{y}$ dimensions. With current technology, interacting with this hyperspectral dataset to perform exploratory analysis, displaying gigapixel elemental maps, and surveying terabyte-scale spectral data is problematic.

We addressed the problem of interacting with terabyte-sized SEM-EDS hyperspectral images by extending the Deep Zoom approach used for large 2D images outside of the field of microanalysis. This approach is based on multi-resolution pyramid representations of 2D images with large pixel counts, and allows for efficient transmission and viewing of images [2]. The initial 2D support was extended to 3D for medical image volumes [3] and to other informative visualizations [4] with an open source project leading to many additional functionalities (see the OpenSeaDragon project [5]). We leveraged the OpenSeaDragon project [5] for building functionality plug-ins. We focused on enabling interactive visual inspections and measurements by supporting real-time interaction with terabyte-sized 3D data, performing off-line re-projections and providing on-demand scale bars and X-ray emission line information overlays. In order to allow further processing and exploration of smaller subsets of terabyte-sized 3D volumes, we added sub-sampling capabilities, executed either client side (i.e in the web browser) or server side, that include a file with provenance information about the sub-sampled data set. To support these functions, we created pyramid representations of the hyperspectral volume that include X-Y and re-projected X-ray spectral views of the dataset, as illustrated in Figure 1 (right). The pyramid set representation corresponding to the X-Y view consists of 4,890,627 input pyramid files in 32,768 folders. The pyramid set for the X-ray spectral view is composed of 4,976,641 output pyramid files in 184,320 folders. Figure 2 shows some of the interactive capabilities accessible in a web browser for the X-ray spectral view. The current capabilities have been deployed in the NIST webspace $[6][7][8]$.

\section{References:}

[1] DE Newbury and NWM Ritchie, J. Anal. At. Spectrom. 28 (2013), p. 973.

[2] Microsoft, "Deep Zoom Silverlight," Microsoft Developer Network (MSDN), 2010. [Online]. http://msdn.microsoft.com/en-us/library/cc645050(v=vs.95).aspx. [Accessed: 27-May-2013].

[3] S Saalfeld, A Cardona, V Hartenstein, and P Tomančák in "The Collaborative Annotation Toolkit for Massive Amounts of Image Data (CATMAID)," (Max Planck Institute of Molecular Cell Biology and Genetics, Pfotenhauerstr. 10801307 Dresden, Germany), 2013.

[4] "Cosmic chronology ChronoZoom," University of California, Berkeley, 2013. [Online]. http://www.chronozoomproject.org/BehindTheScenes.htm. [Accessed: 08-Aug-2013]. [5] "Open Seadragon" Open Seadragon project, 2013. [Online].http://openseadragon.codeplex.com/ [6] http://isg.nist.gov/moche/microscopeWebVisualization.html 
[7] Commercial products are identified in this document in order to specify the experimental procedure adequately. Such identification is not intended to imply recommendation or endorsement by NIST, nor is it intended to imply that the products identified are necessarily the best available for the purpose. [8] The Moche sample was provided by Prof. Michael Notis, Lehigh University and Prof. Aaron Shugar, Buffalo State College. The assistance of Dr. Dale E. Newbury, NIST, is also gratefully acknowledged.

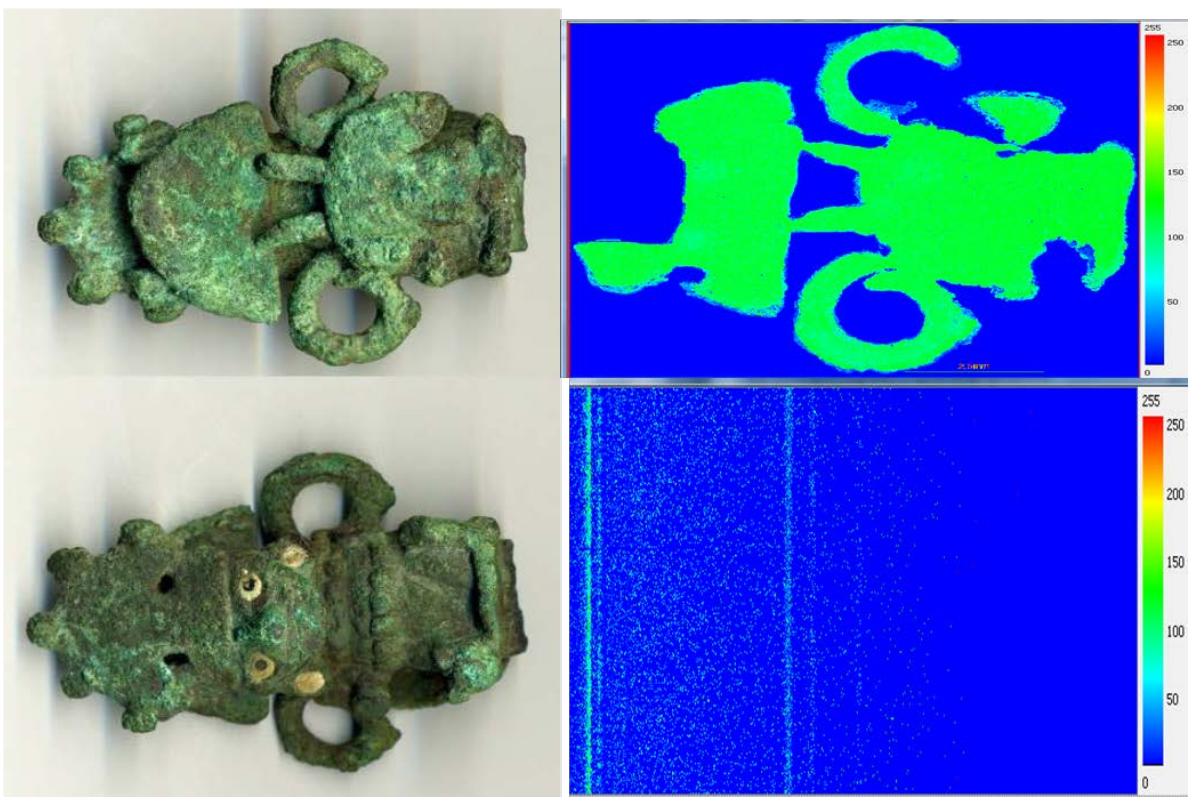

Figure 1. (left) Back and front view photographs of the Moche cast artifact. (right) Spatial X-Y view (top) and SEM-EDS spectral view (bottom) of a cross-sectional surface of the sample. The hyperspectral dataset measures $11520 \times 9,984$ pixels with 2,048 EDS spectral channels per pixel.

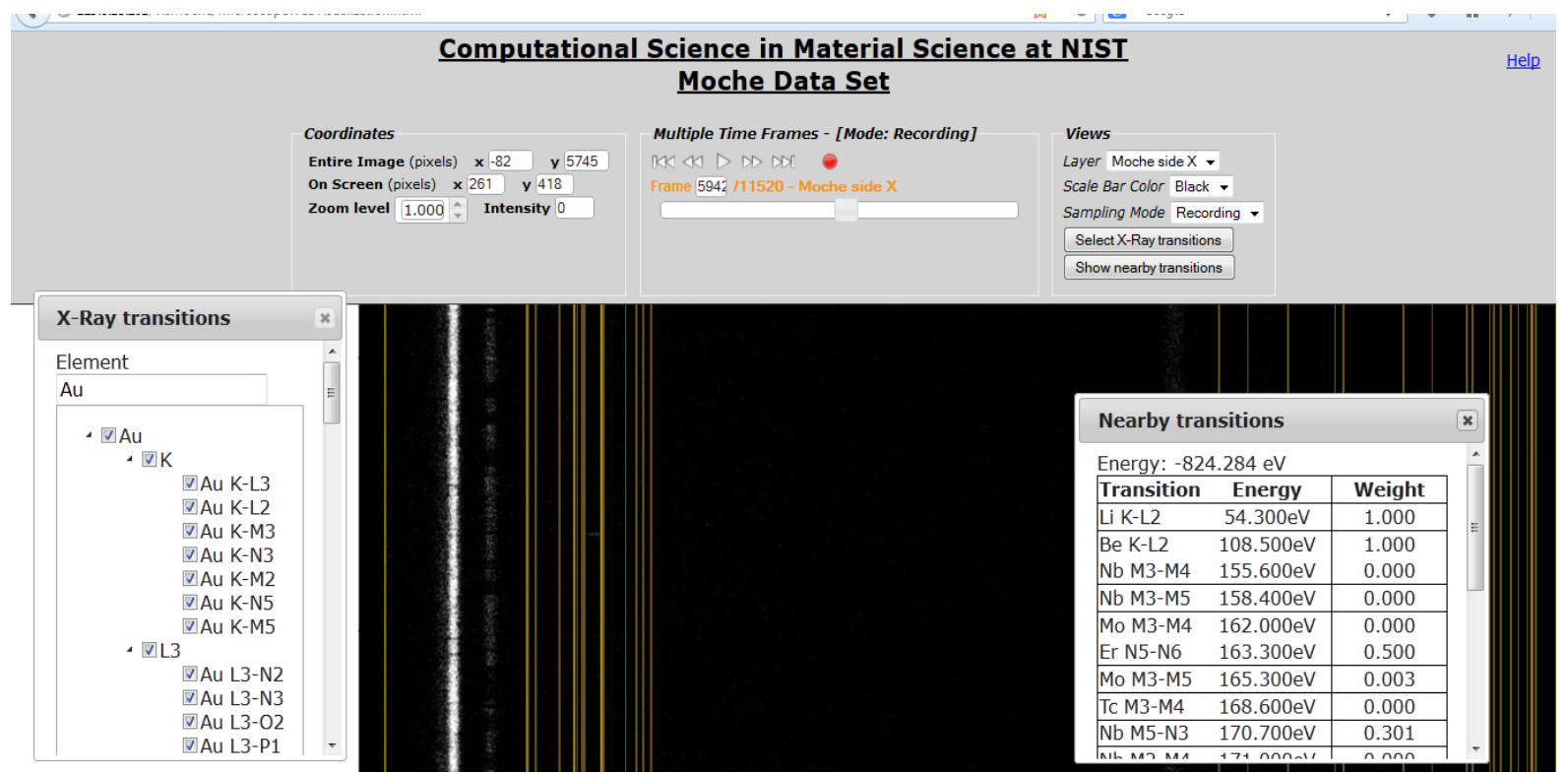

Figure 2. The spectral view of the sample in a web browser with the characteristic spectral line selection dialog (bottom left) and the nearby spectral transition dialog (bottom right). 